\title{
EL ESTATUS EPISTEMOLÓGICO DEL CONCEPTO DE ETNICIDAD
}

\section{THOMAS HYLLAND ERIKSEN}

Traducción: Javier Álvarez Vandeputte*

\section{Introducción: El problema}

Muy a menudo se sostiene, tanto por cientistas sociales como por no expertos, que los miembros de grupos humanos tienen una tendencia "innata" para distinguir entre los de "adentro" y los de "afuera"; para delinear fronteras sociales y para el desarrollo de estereotipos sobre "el otro" con el fin de mantener y justificar estos límites. Si este es el caso, la etnicidad puede concebirse como una característica tan universal para la humanidad como son el género y la edad - a diferencia de fenómenos como la nacionalidad y el nacionalismo, que han sido conceptualizados en la comunidad académica de tal forma que conciernen únicamente al mundo moderno-. (Anderson 1991, Gellner 1983). Marx y Engels, probablemente con razón, señalaban que el sexo, la edad y la distinción nosotros/ellos eran criterios universales de diferenciación. Si, por otro lado, la etnicidad como la conceptualizamos puede ser referida como un producto de un tipo particular de sociedad, por ningún motivo, puede considerarse un fenómeno universal y ahistórico.

La pregunta que se explora en este trabajo, en este momento a modo de boceto, consiste en la ambigüedad de la conceptualización de la etnicidad. ¿Puede ser fructíferamente considerada como una propiedad relacional de cualquier sistema social?, o ¿debe el concepto más bien limitarse a un tipo específico de sociedad histórica, en particular aquellas definidas como sociedades modernas? Para explorar esta pregunta, me basaré en una selección de aportes teóricos y meta-teóricos sobre el análisis de la etnicidad, comenzando con la introducción de Fredrik Barth a su compilación 'Grupos Étnicos y sus fronteras' (Barth 1969)², que en mi opinión representa la defensa más clara de una concepción ahistórica de la etnicidad.

\section{¿Concepto definitivo o espacio definido?}

Mientras que algunos de los argumentos utilizados por Barth pueden parecer obsoletos (por ejemplo, la sección sobre ecología) y otros fueron tal vez menos originales de lo que pudo haber parecido en su momento (cf. por ejemplo, el primer capítulo de 'Sistemas Políticos de Alta Birmania', Leach 1954, o 'La Danza Kalela', Mitchell 1956), su artículo representa una importante ruptura con la visión esencialista sobre etnicidad, antes muy extendida; razón por la que merece un lugar prominente en la historia de los estudios antropológicos sobre etnicidad. En lugar de resaltar la preocupación por las fronteras más que por las "diferencias culturales", que es la idea más influyente en el artículo, voy a indagar en los supuestos epistemológicos subyacentes de su modelo.

* Sociólogo PUC, Magister en Ciencias sociales UCH. Docente adjunto departamento de sociología UCSH.palvarez@ucsh.cl 
El concepto de etnicidad desarrollado por Barth en su "Introducción" podría ser etiquetado a groso modo como naturalista. A pesar de que la mayor contribución original de su ensayo consistió en subrayar que las identidades étnicas se crean desde dentro y no en virtud de las diferencias culturales "objetivas", lo que le otorga la etiqueta de "subjetivista" en algunos sectores, también deja en claro, si bien implícitamente, que los fenómenos étnicos son endémicos de la humanidad y no de algún tipo particular de sociedad. En específico, Barth sitúa la aparición de las distinciones étnicas en la diferenciación dentro de una sociedad y el desarrollo concomitante de formas divergentes de evaluación y limitación de la interacción (Barth, 1969, pp 17-18). Más allá de desvincular definitivamente el concepto de etnia de los conceptos de raza y cultura; quizás la principal contribución epistemológica del artículo de Barth consiste, tal vez, en refinar y relativizar el concepto de sociedad como un fenómeno natural de seres humanos culturales (aunque sin descartar esa definición por completo). Barth muestra que las sociedades pueden ser poli-étnicas y por lo tanto contener grupos delineados y distintivos; que los límites de las sociedades pueden ser no solo relativos, sino también "permeables" en el sentido de que las personas pueden cruzar de forma permanente a otra sociedad (es decir, otro grupo étnico), $y$, por último, que los miembros de un grupo étnico no tienen que compartir todas las caracterizaciones consideradas como definitorias del grupo (un "aire de familia" es suficiente).

Por otro lado, se da por sentado en la mayor parte del texto, el dato de la diferenciación de estatus dentro de una sociedad (en particular, la etnicidad estatutaria) y también se da por sentado, por implicación, la estructura social.
Los sistemas de relaciones que conllevan etnicidad en diversos contextos se consideran implícitamente comparables por Barth (parte final de su ensayo pp. 29ff.) al examen de las variaciones de contexto y sus implicaciones para el análisis. El mecanismo de frontera que define la etnicidad es, en otras palabras, siempre constante y se asume implícitamente como independiente del contexto. De esta manera la etnicidad deviene, en la versión de Barth, un concepto definitorio y en consecuencia un concepto comparativo formal; un puente analítico que no se limita a ningún tipo particular de sociedad o período histórico. La visión de Barth es sostenida por las otras contribuciones que componen el texto 'Los grupos étnicos y sus fronteras' (cf. En especial los trabajos Haaland, Izikowitz' y Knutsson), que tratan principalmente sobre las relaciones interétnicas en sociedades no modernas ni industriales donde la etnia aún no se ha convertido en una fuerza política movilizadora de masas. La mirada sobre la etnicidad presentada en 'Ensayo sobre la antropología del poder y el simbolismo' de Abner Cohen (Cohen 1974a), así como en su importante 'Introducción' a su edición de 'Urban Ethnicity' (Cohen 1974b), difiere de la de Barth. Mientras Barth podría ser etiquetado como un realista moderado, Cohen deja en claro que es un nominalista convencido: para él, la etnicidad no es ni más ni menos que un concepto heurístico útil para dar sentido a procesos históricos delimitados, como la urbanización en África. Así Cohen identifica la etnicidad con los procesos mediante los cuales "algunos grupos de interés explotan parte de su cultura tradicional con el fin de articular funciones organizativas informales que se utilizan en la lucha de estos grupos por el poder" (1974a, p. 91). Algunas páginas más adelante, elabora su noción de etnicidad añadiendo, entre otras cosas, que ésta "implica 
una reorganización dinámica de las relaciones y de las costumbres, y no es el resultado de la conservación o continuidad cultural" (1974a, p. 97). En el análisis de Cohen, la etnicidad aparece como ni más ni menos que una forma de tradicionalismo corporativo, y como tal, limitada a circunstancias propias de la modernidad -presupone la diferenciación institucional, la alfabetización y el estado-.

Sin embargo, tal como Barth, la conceptualización de Cohen también depende de conceptos definitorios ahistóricos y formalistas. A diferencia de Barth, que en parte desarrolló su modelo de frontera para evadir las trampas del funcionalismo estructural (cf. Barth 1966), Cohen abraza la explicación estructural funcionalista, utilizándola de forma explícita como marco general comparativo en el que, en determinadas circunstancias históricas y políticas, emergen los fenómenos empíricos relativos a la etnicidad. Esta lógica también es evidente en los análisis etnográficos de Cohen de los Hausa en Ibadan y los Criollos en Freetown. En otras palabras: mientras que en Barth la etnicidad se presenta como un fenómeno social natural, para Cohen es relegada a la condición de un fenómeno histórico contingente -esto es, como parte del espacio definido, y como parte de la sociedad analizada. Una pregunta a la que tendremos que volver es, obviamente, si los dos autores tienen el mismo fenómeno en mente cuando hablan de etnicidad.

\section{Constructivismo y relatos históricos}

El texto 'Ethos e identidad' de Arnold Epstein (Epstein, 1978) marcó un cambio decisivo de enfoque en el estudio de la etnicidad en antropología social. Mientras que Barth y Cohen estaban implícitamente de acuerdo en que la etnicidad se distingue mejor como una forma de acción política, Epstein repara en la dimensión de identidad en la etnicidad más que en la dimensión política, y se centra en gran medida en la psicología social, en particular en el trabajo de Erik H. Erikson, al sostener que "necesitamos complementar las perspectivas sociológicas convencionales prestando mayor atención a la naturaleza de la identidad étnica" (Epstein, 1978, p. 5). Sin embargo, contrariamente a lo que cabría temer de la declaración programática de Epstein; desde el principio en sus tres estudios, él no abandona las "perspectivas sociológicas convencionales". De una manera que se asemeja, probablemente no por accidente, al trabajo de Victor Turner, Epstein combina un problema cognitivista con el significado simbólico y una base de análisis sociológico en lo que es uno de los estudios más profundos de la identidad étnica a la fecha. También es claro que Epstein, como Cohen, considera en gran medida la etnicidad como una creación de la modernidad. Sus casos de estudio, que van desde el Cinturón de Cobre a Melanesia y la diáspora judía contemporánea, indican que la formación de las identidades étnicas, y esa comunicación socialmente organizada y orquestada de la distinción cultural que constituye la etnia, ocurren en situaciones de rápido e incontrolable cambio social -es decir, el impacto del mundo moderno El enfoque psicológico social de Epstein podría ser complementario a la visión sociológica de Cohen, al centrarse en los aspectos no utilitarios y no funcionales de la creación individual de significado en el proceso de etnogénesis, pero que lidian esencialmente con el mismo tipo de situación social.

Una cuarta posición podría describirse como enfoque social constructivista. Inspirada en 
las tres perspectivas 'clásicas' descritas, pero desde una posición reflexiva, los representantes de esta posición son más explícitos en disociar etnia de "raza" y "cultura", centrándose en las formas en que las identidades y las fronteras étnicas son históricamente arbitrarias y son la construcción de miembros de las élites que buscan poder político y/o ganancias materiales - o, la construcción de un grupo dominante que busca intimidar a los grupos dominados mediante la imposición de etiquetas étnicas-. Cada uno a su manera, Eugeen Roosens (1989) y los editores de la monografía de la ASA titulada 'History and Ethnicity' (Chapman et al 1989) representan una corriente que hace hincapié en la importancia del "punto de vista del nativo" en el desarrollo de las identidades étnicas. Sus puntos de vista son, quizás, derechamente "subjetivistas" (a diferencia de Barth, que combina factores subjetivos y objetivos), ya que consideran que los grupos étnicos son posibles, pero no los productos necesarios de los esfuerzos creativos bajo determinadas circunstancias históricas. En su opinión, las explicaciones culturalistas sobre las etnias son tan invalidas como aquellas centradas en las razas, ya que la formación de la identidad étnica remite a la apropiación más o menos al azar de supuestos rasgos culturales y la sobredeterminación [overcommunication] de esos rasgos. El grado de capacidad explicativa otorgado a los factores sociales dentro de esta matriz de exploración varía; Benedict Anderson, por ejemplo, podría claramente ser visto como un constructivista, aunque él insiste en la necesidad de fuerzas tecnológicas objetivas en la aparición de las identidades étnicas (o nacionales).

Finalmente está la aproximación histórica a los estudios de etnicidad. Este punto de vista comporta un creciente interés en el análisis histórico dentro de la antropología, cuyo inicio se remonta al momento en que los marxistas y los auto denominados neo-marxistas estaban de moda y que continua hasta el día de hoy. John y Jean Comaroff, en 'Etnografía y la Imaginación Histórica' (Comaroff y Comaroff 1992), hacen una declaración sobre su posición cuando afirman, en una seductora comparación entre etnicidad y totemismo, que "contrariamente a la tendencia a verla como una función de los lazos primordiales, la etnicidad siempre tiene su génesis en fuerzas históricas específicas, fuerzas que son a la vez estructurales y culturales" (p. 50). El mundo moderno, mediante la monetarización, se vuelve comparable en función del valor; Pero también hace que las diferencias culturales se vuelvan comparables y por lo tanto estimula la aparición de grupos étnicos - en palabras de Comaroff y Comaroff, "la etnicidad tiene su origen en la incorporación asimétrica de grupos estructuralmente diferentes en una misma economía política" (p. 54). Este tipo de aproximación es seguida por otros investigadores, por ejemplo, en el importante trabajo de John Peel sobre Nigeria (Peel 1989), donde se argumenta, contra Abner Cohen, que aunque la etnia Yoruba surgió históricamente como respuesta a los cambios sociales impuestos por el colonialismo, ésta emergió bajo circunstancias específicas que incluían importantes dimensiones culturales y que la etnicidad Yoruba no puede explicarse satisfactoriamente sin una consideración de los factores culturales e históricos. Este argumento también podría ser dirigido contra las posiciones social-constructivistas más extremas, así como en contra de la explicación estructural-funcionalista de Cohen: Peel muestra que, aunque las identidades étnicas pueden aparecer como 
invenciones, éstas no son, ciertamente, invenciones arbitrarias; y que la causalidad histórica limita drásticamente no sólo la gama de opciones para la construcción deliberada de identidades, sino también su forma.

\section{El problema del mapa-territorio}

La discusión conducida hasta ahora puede parecerse a los antiguos debates meta teóricos sobre el concepto de etnicidad, sobre todo el viejo debate subjetivismo-objetivismo y primordialismo-instrumentalismo. Sin embargo, estas distinciones ya no parecen dar cuenta de las principales diferencias teóricas. Las posiciones descritas podrían ser descritas como naturalistas (Barth), estructural-funcionalistas (Cohen), mentalistas (Epstein), constructivistas (Roosens, Chapman et al.), e historicistas (Comaroff, Peel), y podrían ser dispuestas en un continuo que va desde el subjetivismo extremo (las posiciones constructivistas) a un objetivismo moderado (Comaroff). Sin embargo, para ser justos, todas estas posiciones coinciden en que la etnicidad implica una combinación contingente de factores subjetivos y objetivos; variando el peso que se le otorga a cada dimensión.

Respecto al tema de la cultura como una fuerza determinante de la etnicidad vs cultura como fantasía ideológica reificada; las visiones más inclinadas a la última son las de Epstein y Barth; pero todos los autores examinados se han distanciado de la idea weberiana de que las identidades étnicas son "naturales", y pueden, por lo tanto, ser tratadas como variables independientes. Cuando Barth es etiquetado de naturalista, no es más que para llamar la atención de su modelo formal empíricamente vacío de etnicidad -su concepto de etnicidad es aclaratorio, pero no un agente de causalidad.

Los debates sobre lo objetivo vs subjetivo y lo instrumental vs primordial parecen haber sido superados. El problema más apremiante con respecto al estatuto epistemológico del concepto de etnicidad, parece ser la relación entre la etnicidad como propiedad de todas y cada una de las relaciones intergrupales, y la etnicidad como el producto de un tipo particular de situación histórica.

Con el fin de desentrañar los diferentes aspectos de esta cuestión, será útil mirarlo en términos de las conceptualizaciones actuales. En otras palabras: ¿Hablan los diferentes analistas de lo mismo cuando dicen "etnicidad"?

\section{Los niveles de investigación}

Para los antropólogos el dato fundamental de la etnicidad es la aplicación de una distinción sistemática entre "nosotros" y "los otros". Un extraordinario número de trabajos antropológicos sobre las más diversas sociedades indican que esta distinción es, de hecho, universal. Estudios como el de Middleton sobre la brujería Lugbara o el de Leach sobre la sociedad Kachin, indican que la distinción nosotros-ellos es una característica perenne de los grupos humanos. Aparentemente, la etnicidad surge en el momento en que estos grupos entran en contacto con otros (En retrospectiva, podemos quizás añadir: La etnia entonces aparece al menos en los ojos del que mira). Además, como Hirschfeld (1988) ha argumentado, incluso los niños más pequeños parecen capaces de distinguir más o menos espontáneamente entre "clases" de personas en otras palabras, los seres humanos parecen 
estar genéticamente predispuestos a este tipo de distinciones. La mirada de Epstein de la etnicidad resuena con esta perspectiva de la humanidad. Epstein considera que hay una necesidad psicológica fundamental que moviliza en la búsqueda de una identidad social sólida, segura y más o menos limitada; y conecta esta necesidad, en circunstancias históricas particulares, con la formación de identidades étnicas

¿Deben las identidades sociales, vistas como etiquetas contrastivas y clasificaciones mutuamente excluyentes, ser consideradas siempre y necesariamente como étnicas? ¿Es necesario que la interacción posea alguna otra característica para que una configuración particular sea etiquetada como "étnica"? Esto me parece que es el corazón de la cuestión. La respuesta de Comaroff es "sí", y clasifican, por ejemplo, la relación Nuer-Dinka como totémica, no interétnica, ya que es la expresión de un "tipo diferente de conciencia" (1992, p.55). La etnicidad ocurre cuando la percepción de las diferencias culturales provoca una diferencia social; eso hay que darlo por sentado. Sin embargo, una definición comparativa útil requiere mayor precisión, y cabe señalar aquí que todos los analistas citados llegan a definiciones heurísticas útiles; aunque se trata de definiciones divergentes.

La siguiente, pregunta inevitable debe ser: ¿Qué conceptualización de etnicidad es la más útil en antropología comparada? El concepto formal, empíricamente vacío, sostenido por Barth permite un rango amplio de comparaciones de los fenómenos étnicos, ya que teóricamente incluye las relaciones Yanomami-Cholo, noruego-Sami, Fur-Baggara, negros-británicos blancos, así como muchas otras relaciones que tienen poco en común aparte de la reproducción social de fronteras étnicas. Al menos en el caso de la relación Fur-Baggara, la modernización parece no haber influido en grado significativo desde el tiempo en que Haaland (1969) desarrolló su trabajo de campo (Considerando el análisis histórico de la etnogénesis de Sudán de O'Brien de 1986).

El resultado de comparaciones a este nivel de generalidad está limitado a conclusiones altamente abstractas como: La etnicidad implica tanto dicotomización y complementarización - tanto contraste como comparación(Eidheim 1969); Los individuos pueden cruzar las fronteras étnicas sin perturbar su funcionamiento básico (Haaland 1969); la relación entre la cultura y la etnicidad no es del tipo uno-a-uno (Blom 1969), y en un nivel aún más general: la etnicidad implica hacer diferencias comparables. Este tipo de comparación, que corre el riesgo de comparar peras con manzanas, claramente tiene su valor, ya que permite formular preguntas más específicas a nuestra evidencia. Por ejemplo, las diversas situaciones interétnicas pueden ser comparadas en términos de apertura versus cierre, dominación versus equidad, y la correlación entre origen étnico y división del trabajo.

Lo que esta conceptualización de la etnicidad no facilita, es una mejor comprensión de la aparición de determinadas identidades étnicas y relaciones interétnicas particulares. Eso, sin embargo, se puede lograr a partir de la investigación de esas sociedades particulares; el punto es que un concepto formal de etnicidad, multi-abarcador, puede servir de punto de partida o de posición estratégica (brigedhead) para la investigación de los rasgos singulares de una sociedad en particular (véase Eriksen, 1992, capítulos 1-2). 
En este punto, se podría objetar que esta maniobra supone una comparación brutal y profundamente positivista de contextos que no pueden ser significativamente comparados. Una objeción, por ejemplo, es que la etnicidad no existe de la misma manera en sociedades individualistas y en sociedades holísticas. Esta sería probablemente la posición argumentativa de Dumont de haber participado en este debate: en otro lugar (p.e. Dumont 1983), él ha criticado a los científicos sociales europeos post-tönniesianos por suponer que la "acción lógica" europea y la metafísica individualista son universales.

\section{Reflexividad moderna}

En consonancia con este razonamiento, se ha argumentado que no sólo la etnicidad debe en gran medida ser considerada como un constructo, sino que es, en muchos casos, la construcción del analista (Fardon 1987, Ardener 1989). Ya antes, Aidan Southall (1976) ha argumentado que los "Nuer" y los "Dinka", en tanto que grupos étnicos, eran más o menos las construcciones de Evans-Pritchard y Lienhardt, respectivamente: Nadie posiblemente podría definirse a sí mismo como miembro de esos "pueblos". Fardon y Ardener argumentan el mismo punto con respecto a dos pueblos de África Occidental, la Chamba y Kole, respectivamente. Es probable, según Fardon que los Chamba invocaran la distinción "nosotrosellos" en la época precolonial, pero éstas eran más fluidas, situacionales y superpuestas, y, en general, menos clara que las distinciones étnicas generadas cuando el limite étnico ya está trazado.
La historia del nacionalismo puede dar pistas sobre el origen de la actual concepción antropológica de grupo étnico. De hecho, el concepto de "tribu" así como el de "grupo étnico" son conceptualmente parientes cercanos del concepto europeo de nación, entendido éste como unidad soberana culturalmente homogénea. El concepto de grupo étnico aparece así como un hijo del nacionalismo - en cuyo caso su uso podría quedar limitado para el estudio de las sociedades no modernas-. Sin embargo, al menos en el artículo de Fardon sobre los Chamba estos finalmente han llegado a considerarse a sí mismos como un grupo étnico en lo que parece, más o menos, como una reminiscencia de la conceptualización antropológica de los grupos étnicos. De hecho, han recogido en parte este uso antropológico, y por otra parte han sido influenciados por procesos sociales de transformación en una dirección que permite el surgimiento de grupos étnicos (en este sentido) - en una palabra, sociedades capitalistas letradas con un sistema educativo formal-. Así, de manera epistemológica y metodológicamente confusa, los llamados informantes se han apropiado en los últimos años de los conceptos y análisis de los antropólogos y han convertido a estos conceptos en material y datos empíricos.

La situación parece ser un colapso de parámetros según e término de Ardener) donde nuestros conceptos de cultura y etnicidad, antes conceptos definitorios por excelencia, colapsaban en el espacio definido; en lugar de servir como puntos de apoyo conceptuales, pasanban a formar parte de la realidad social que necesitaba ser indagada. 
Esto, evidentemente, es el tipo de etnicidad de la que habla Roosens en sus análisis de los indios Hurones y los Luba de Kasai (Roosens 1989). Los hurones, en particular, han formado de manera consciente "una cultura" y una identidad social que encaja perfectamente con la visión contemporánea de las minorías y la política de las minorías, lo que les permite perseguir objetivos políticos dentro del contexto del Estado canadiense -circunstancia que debe mucho a los conceptos y modelos antropológicos-. Esta opción no estaba presente para los Fur de Haaland's, aunque parece claro que sostenían nociones ideológicas acerca de su distinción cultural vis-à-vis (frente a frente) con respecto a los Baggara.

\section{Etnicidad y difusión}

Estudios como "Creando etnicidad" de Roosens indican lo fructífero de un concepto de etnicidad históricamente situado. En este libro, que presenta un rango amplio de situaciones interétnicas, el autor ha acuñado un concepto de etnicidad que abarca tanto las dimensiones sociopsicológicas y de reflexividad de la etnicidad, así como también las dimensiones políticas. Al mismo tiempo, Roosens sostiene que "entre los seres humanos, cualquiera que sea su tradición cultural, son muy deseables una serie de bienes y valores materiales cuya producción se originó en la sociedad occidental". En el análisis de Roosens, que abarca desde los hurones de Quebec a las minorías en Bélgica, la etnicidad, que no necesariamente se ha difundido de manera directa, aparece como una especie de respuesta a la difusión de ciertas dimensiones vitales de la modernidad.

Un punto de vista más explícitamente difusionista es sostenido por Peter Worsley (1984), quien señala que sin duda no deja de tener interés académico que los tamiles de Sri Lanka, antes del surgimiento del movimiento Tamil separatista, llamado Eelam; debe haber visto los noticias sobre Cisjordania y la lucha palestina.

Adoptar esta perspectiva en los estudios comparativos de etnicidad tiene consecuencias epistemológicas. Si nos centramos en la autoconciencia étnica, en la forma en que se propaga por los medios de comunicación o la migración, o como se desarrolla través de procesos históricos similares en sociedades diferentes, entonces la forma antropológica clásica de comparación cuasi experimental entre sociales discretas y supuestamente aisladas entre sí debe desecharse. A mi juicio, es evidente que este método tendrá que ser abandonado. En este mundo sin fisuras, ya no es factible mantener variables constantes en el cuasi-experimento de comparación antropológica: hay demasiada distorsión para que este estilo de comparación sea viable. En su lugar, una estrategia sensata de comparación debe trazar las interconexiones entre las sociedades y explicar sus expresiones locales. Estas interconexiones incluyen aspectos "objetivos" y "subjetivos" de la etnicidad, y esta ya sea que se estudie como identidades contrastantes o como organización política, debe ser vista en el mundo de hoy en relación con los procesos de globalización. Esto no implica que la etnicidad sea, "ontológicamente", nada más que un subproducto del capitalismo, del Estado y de los medios de comunicación masivos, sino que sus expresiones son al menos hoy contingentes a tales parámetros.

Este concepto de etnicidad históricamente vinculado nos permite comparar las modernidades en un marco conceptual más amplio, 
que no puedo presentar aquí (véase Eriksen 1993:Cp 8). Algunas cosas, sin embargo, se pueden sugerir, aunque sea brevemente.

El melanesista Edvard Hviding ha sostenido (1993) que la tendencia general hacia el parentesco cognaticio entre los isleños de Solomon ha sido en los últimos años desafiada por una preocupación por el parentesco unilineal, especialmente del tipo patrilineal. Hviding explica esto como una expresión de los intereses políticos a nivel de clan para el logro de los derechos territoriales y de organización corporativa. Este desarrollo es muy similar al desarrollo descrito por Fardon (1987) en su estudio de la etnogénesis de los Chamba: es contingente a la modernidad, el colonialismo, la alfabetización, la propiedad privada, el individualismo y varios otros parámetros que, tras su introducción, producen contextos discretos comparables a lo largo de nuevas líneas. En otras palabras, la posición histórica de la etnicidad tal como la conocemos debería incluir variables contextuales, así como los hechos desnudos de las distinciones étnicas, haciendo posible comparar rasgos empíricos de las sociedades, no simplemente sus características formales.

\section{Observaciones finales}

En lugar de enmarcar la cuestión sobre la naturaleza de la etnicidad en algún lugar, dentro o alrededor, de la familiar dicotomía instrumentalismo/primordialismo, he optado por discutirlo en términos conceptuales. Esto porque la etnicidad es ante todo un concepto y no un fenómeno natural. Como concepto es que existe en (al menos) dos niveles, el del analista y el del nativo. ¿Es posible - o incluso deseable - mantenerlos separados? Si lo hacemos, el concepto conservará su potencial comparativo de amplio alcance, y si no lo hacemos, nos permitirá describir los contextos locales de una forma más cercana a la experiencia.

La etnicidad puede ser vista como un fenómeno social universal y puede ser vista como una construcción cultural moderna. Puede conceptualizarse como una clase de organización política informal (Cohen), como uno de los aspectos de la identidad personal implicado en la asignación contrastiva de etiquetas mutuamente excluyentes (Epstein), como la apropiación reflexiva de un "patrimonio cultural", la historia y los derechos políticos concomitantes (Roosens), como producto del colonialismo y del capitalismo (Comaroff, Fardon), o como un mecanismo funcional de fronterización que separa grupos endogámicos (Barth). Si, por ejemplo, el foco de análisis está en la autoidentidad [self-identity] anclada en la reflexión sobre la tradición, se requiere de un concepto de etnicidad históricamente situado. $\mathrm{Si}$, por el contrario, la investigación pretende trazar, por ejemplo, aspectos de los procesos básicos de interacción o de formación de la identidad social, se necesita una conceptualización más abarcadora y formal. Por lo tanto, la pregunta no se enmarca en "qué es etnicidad", sino "¿cómo conceptualizar más fructíferamente la etnicidad?" Sospecho que la mayoría de las controversias sobre las definiciones provienen de una inadecuada distinción al respecto: de una preocupación implícita (o explícita) sobre las "esencias" y una inocencia positivista con respecto al estatus ontológico de nuestros conceptos. La mayoría de ellos, por lo menos en las disciplinas sociales, tienen una vida útil bastante corta y no hay razón para creer que el concepto de etnicidad actual, todavía útil, seguirá estando con nosotros en unos años 
más. Como he insinuado, la frontera entre la etnicidad como concepto popular y como herramienta analítica está actualmente bajo tensión - los nativos tienen sus propias teorías antropológicamente informadas de etnicidad - y parece que el concepto, hasta ahora un concepto definitorio, está a punto de colapsar, como Ardener lo habría dicho, hacia un espacio

\section{Notas}

${ }^{1}$ Conferencia, Ámsterdam ("La Antropología de la Raza"), diciembre de 1993. Publicado en Cuadernos de antropología (Liubliana, Eslovenia) en 1996.

2 El texto, ya clásico, "Los grupos étnicos y sus fronteras" es una

\section{Referencias bibliográficas}

Anderson, B. (1991 [1983]). Imagined Communities. Reflections on the Origins and Spread of Nationalism. London: Verso.

Ardener, E. (1989). The Voice of Prophecy and other essays, ed. Malcolm Chapman. Oxford: Blackwell.

Barth, F. (1966). Models of Social Organization. Occasional Papers, (23).

(1969) Introduction. En Barth, F. (ed.), Ethnic Groups and Boundaries. The Social Organization of Culture Difference, (pp. 9-38). Oslo: Scandinavian University Press.

Blom, J-P. (1969). Ethnic and cultural differentiation. En Barth, F. (ed.), Ethnic Groups and Boundaries. The Social Organization of Culture Difference, (pp. 75-85). Oslo: Scandinavian University Press.

Chapman, M., McDonald, M. \& Tonkin, E. (1989). Introduction - history and social anthropology. En Tonkin, E., McDonald, M. \& Chapman, M. (eds.), History and Ethnicity, (pp. 1-21). London: Routledge.

Cohen, A. (1974a). Two-Dimensional Man. London: Tavistock. (1974b). Introduction: The lesson of ethnicity. En Cohen,

A. (ed.), Urban Ethnicity, (pp. ix-xxii). London: Tavistock.

Comaroff, J. \& Comaroff, J. (1992). Ethnography and the Historical Imagination. Boulder: Westview.

Dumont, L. (1983). Essais sur l'individualisme. Paris: Seuil.

Eidheim, H. (1969). When ethnic identity is a social stigma. En Barth, F. (ed.), Ethnic Groups and Boundaries. The Social Organization of Culture Difference, (pp. 39-57). Oslo: Scandinavian University Press.

Epstein, A.L. (1978). Ethos and Identity: Three Studies in Ethnicity. London: Tavistock

Eriksen, T. H. (1992). Us and Them in Modern Societies: Ethnicity and Nationalism in Trinidad, Mauritius and Beyond. Oslo: Scandinavian University Press. definido. Quizás sea el momento de prepararnos para reemplazar nuestros conceptos de etnicidad por términos como tradicionalismo, culturalismo, cultura politizada y organización política informal en un número creciente de casos. Tales conceptos crearán un nuevo espacio definido, posiblemente uno más beneficioso para la investigación. O quizás no.

compilación de trabajos de distintos autores a cargo de Frederick Barth. Es en la introducción donde él desarrolla y plasma su perspectiva sobre la etnicidad. Con el tiempo se ha vuelto la sección más importante y citada del texto. Nota del traductor.
(1993). Ethnicity and Nationalism: Anthropological

Perspectives. London: Pluto.

Fardon, R. (1987). 'African ethnogenesis': Limits to the comparability ethnic phenomena. En Holy, L. (ed.), Comparative Anthropology, (pp. 168-188). Oxford: Blackwell.

Gellner, E. (1983). Nations and Nationalism. Oxford: Blackwell.

Haaland, G. (1969). Economic determinants in ethnic processes. En Barth, F. (ed.), Ethnic Groups and Boundaries. The Social Organization of Culture Difference, (pp. 58-74). Oslo: Scandinavian University Press.

Hirschfeld, L. (1988). On acquiring social categories: cognitive development and anthropological wisdom. Man, 23, 611-38.

Hviding, E. (1993). Personal communication.

Izikowitz, K. G. (1969). Neighbours in Laos. En Barth, F. (ed.), Ethnic Groups and Boundaries. The Social Organization of Culture Difference, (pp. 135-48). Oslo: Scandinavian University Press.

Knutsson, K. E. (1969). Dichotomization and Integration. En Barth, F. (ed.), Ethnic Groups and Boundaries. The Social Organization of Culture Difference, (pp. 86-100). Oslo: Scandinavian University Press.

Leach, E. R. (1954). Political Systems of Highland Burma. London: Athlone.

Mitchell, J. C. (1956). The Kalela Dance. Rhodes-Livingstone Papers, (27).

O'Brien, J. (1986). Toward a reconstitution of ethnicity: Capitalist expansion and cultural dynamics in Sudan. American Anthropologist, 88, 898-906.

Roosens, E. E. (1989). Creating Ethnicity. London: SAGE.

Southall, A. (1976). Nuer and Dinka are people: ecology, ethnicity and logical possibility. Man, 11(4), 463-91.

Worsley, P. (1984). The Three Worlds: Culture \& World Development. London: Weidenfeld \& Nicholson. 